\title{
Development of Hybrid Three-axis Load Sensor
}

\author{
Kenta Watanabe, ${ }^{1,2}$ Kazuhiro Hara, ${ }^{1}$ and Shigeru Toyama ${ }^{2 *}$ \\ ${ }^{1}$ School of Engineering, Tokyo Denki University, 5 Senju Asahi-cho, Adachi-ku, Tokyo 120-8551, Japan \\ ${ }^{2}$ Research Institute, National Rehabilitation Center for Persons with Disabilities, \\ 4-1 Namiki, Tokorozawa-shi, Saitama 359-8555, Japan
}

(Received October 22, 2019; accepted January 27, 2020)

Keywords: three-axis load sensor, shear force sensor, pressure sensor, flexible sheet sensor, sensor for persons with disabilities

A thin and flexible sheet-type three-axis load sensor has been developed by hybridizing two different types of sensors, namely, a commercial sheet-type pressure sensor and a shear force sensor that we previously developed. The total thickness of the sensor was $0.9 \mathrm{~mm}$. We have also developed a control circuit and a computer program for the sensor. Although seven signal lines were required in the original sensor configuration, the number of signal lines could be reduced to six by devising the circuit. The sensor response was as linear as each of the component sensors, and the responses of each of the three axes were nearly independent, without particular combination problems.

\section{Introduction}

Persons with physical disabilities use various instruments in contact with the body, such as wheelchairs, artificial hands, and artificial legs. Contact between these instruments and the body often continues for a long time, and shear force and pressure act on the contact surface. For a long time, these forces have been considered to be factors that cause blood flow occlusion and wounds on the skin of the contact surface. ${ }^{(1-3)}$ Pressure sores originating from such wounds are a global problem in the medical and rehabilitation fields. Pressure sores occur when external blood pressure on a part of the human body causes the blood flow in the tissue between the skin and the bone to stop or decrease. If this condition persists, the tissue suffers from irreversible ischemic damage. ${ }^{(4)}$ Therefore, attempts have been made to evaluate how much pressure or shear force is applied to the skin. Bennett et al. ${ }^{(5)}$ reported that blood flow is halved when a shearing force of $0.01 \mathrm{MPa}$ is applied to the palm, and that the effect of pressure is approximately doubled. After this discovery, several research groups tried to evaluate the shearing force applied to the buttocks placed on a wheelchair. ${ }^{(6)}$ For example, Kobara et al. used a kind of force plate to measure the total shear force applied to the entire seating surface of a wheelchair. ${ }^{(7)}$ Recently, we developed a shear force sensor to evaluate the local shear force in different postures, and found that regardless of posture, a backward-directed shearing force occurred below the ischial tuberosity in seated positions. ${ }^{(9)}$ Such an experiment was possible because the sensor was thin and flexible, so that it could be installed between the cushion sheet 
of the wheelchair and the buttocks of the subject. We need a three-axis thin and flexible sensor to further develop the experiment.

Apart from pressure ulcer research, thin and flexible sensors have other uses. For example, they can be inserted into the prosthetic socket to evaluate the force between the inner surface of the socket and the stump of an amputee. Sanders and Daly conducted an experiment by attaching a triaxial force sensor to a prosthesis. ${ }^{(10)}$ Although it was a pioneering work, they had to attach the bulky sensor by drilling a hole in the prosthesis socket. It is possible to acquire data in a more natural state if the sensor can be attached to the prosthetic in a nondestructive way.

Recently, various small three-axis sensors have been developed. Typical sensors with different operating principles are strain-gage-based micro-electromechanical system (MEMS) sensors, ${ }^{(11-13)}$ light-detection-type sensors, ${ }^{(14,15)}$ capacitive sensors, ${ }^{(16,17)}$ and sensors using conductive rubber. ${ }^{(18)}$ However, some of them are not necessarily thin and flexible even if they are tiny.

Thus far, we have developed a unique flexible sheet-type shear force sensor that uses a liquid electrolyte. ${ }^{(9,19)}$ We have already been conducting applied experiments using this sensor with collaborative research studies..$^{(20,21)}$

One of the advantages of using a liquid electrolyte is that the contact interface between the electrode and the electrolyte is stable when an external force is applied. When the conductive rubber and electrode come into contact with each other, peeling at the interface due to external force may occur if the adhesiveness is insufficient. Moreover, the sensor is flexible because its constituent material is liquid. Because the liquid electrolyte is an incompressible fluid, volume changes due to pressure hardly occurs; an advantage of biaxial shear force sensors is that they are not easily affected by pressure. However, it is not easy to add a function to detect pressure. Therefore, we decided to develop a triaxial force sensor by combining a shear force sensor with a conventional pressure sensor.

Furthermore, for applications, it is important that not only the sensor itself but also the cable connected to the sensor can be extended for a long distance while being flexible and flat. Since a liquid-based shear force sensor measures current instead of potential, it is robust to not only electrostatic noise but also stray capacitance, so it is also possible to extend the cable without using a preamplifier.

As shown in Fig. 1, the three-axis load sensor has a structure where a shear force sensor and a pressure sensor are laminated. The shear force sensor is composed of upper and lower flexible electrode substrates and a rubber ring. The unique point is that an electrolyte solution is filled in the minute space between the upper and lower electrode substrates and the rubber ring. The upper electrode substrate has one electrode (center electrode) at the center, and a total of four electrodes (peripheral electrodes) are point-symmetrically arranged on the lower electrode substrate.

Figure 2 shows a cross-sectional view of the three-axis load sensor. In the state where no external force is applied to the sensor, the center electrode and each peripheral electrode are equidistant. However, when applying a shear force to the sensor, the upper and lower electrode substrates horizontally shift from each other. As a result, the distances between the center 


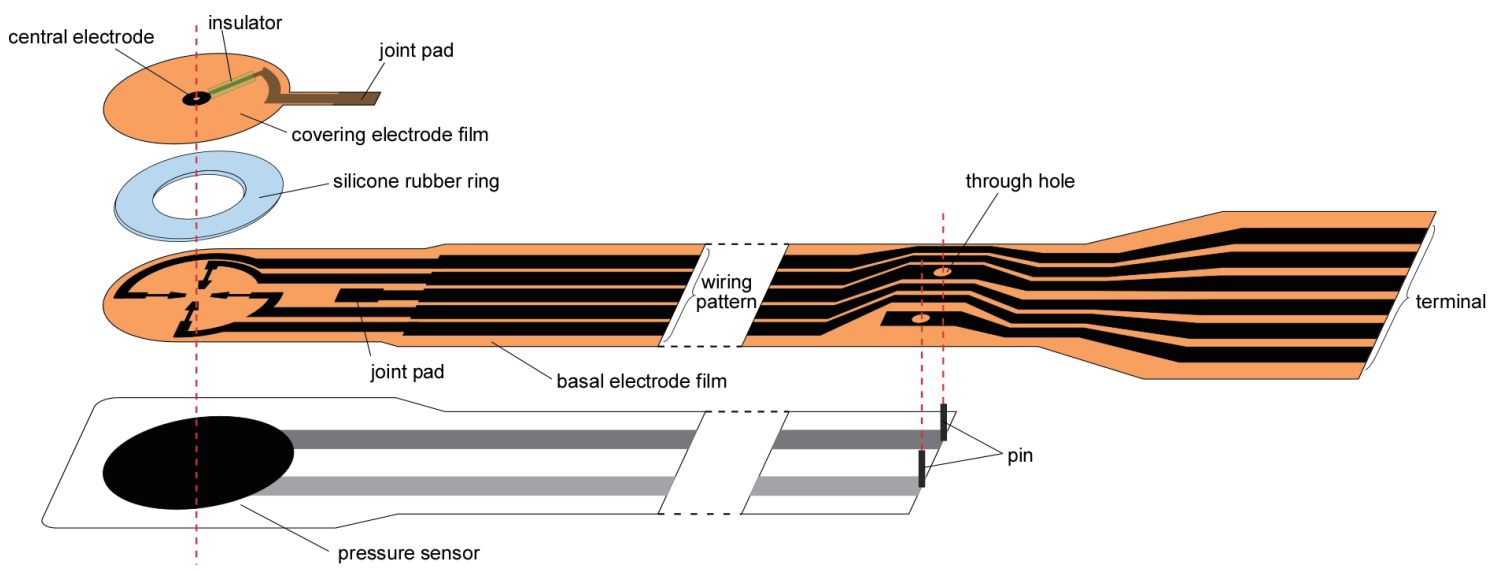

Fig. 1. (Color online) Structure of three-axis load sensor.

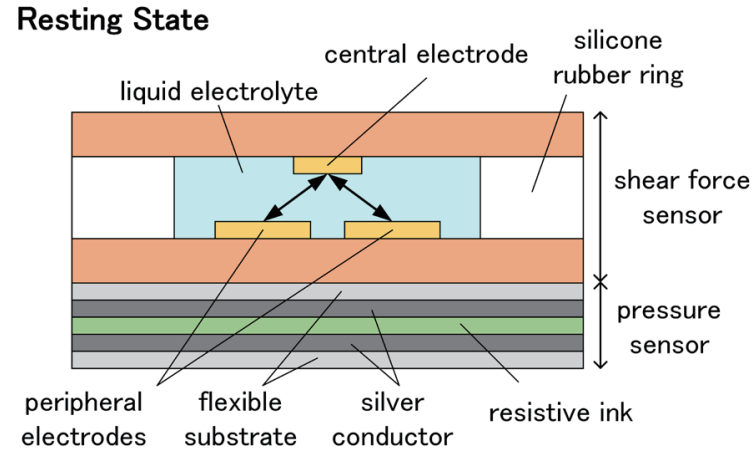

(a)

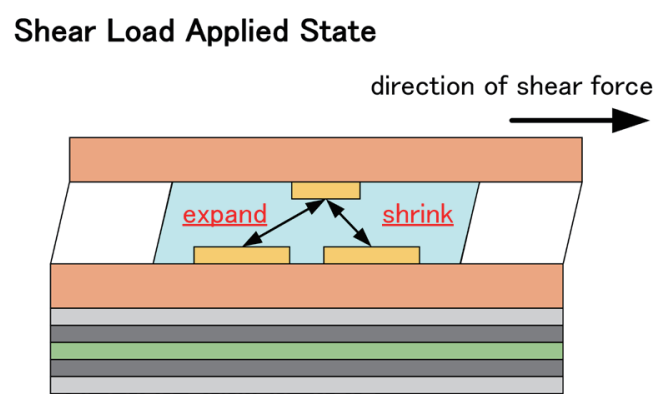

(b)

Fig. 2. (Color online) Sectional view of three-axis load sensor. (a) Resting and (b) shear-load-applied states.

electrode and the peripheral electrodes become asymmetric, and the conductance between the electrodes changes. Therefore, by measuring the current flowing between the center electrode and each peripheral electrode, we can estimate the shear force applied to the sensor. On the other hand, when pressure is perpendicularly applied, the response of the pressure sensor can be obtained.

However, the total number of wiring cables is seven, corresponding to the total number of shear force sensor and pressure sensor electrodes. In practice, it is desirable to reduce the number of cables and to make the entire cable slim. Therefore, we reduced the number of cables to six by devising a circuit.

\section{Materials and Methods}

\subsection{Fabrication of three-axis load sensor}

The shear force sensor was fabricated by referring to our previous article. ${ }^{(9)}$ The flexible electrode substrates were prepared by performing electron beam evaporation in the order of

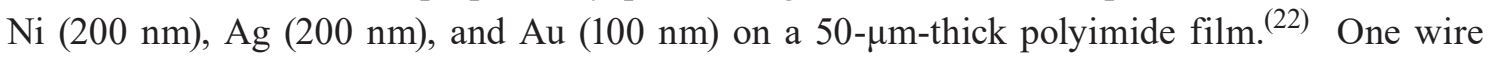


protruding from the upper electrode substrate was soldered to the pad of the lower electrode substrate. The upper and lower electrode substrates and the silicone rubber ring $(0.5 \mathrm{~mm}$ thick) were bonded with an adhesive while performing precise alignment. A liquid electrolyte prepared by dissolving lithium chloride $(350 \mathrm{mM})$ in ethylene glycol was sealed in the space between the upper and lower electrode substrates and the rubber ring. An acrylic modified silicone adhesive (AX-016, Cemedine Co.) was used for the insulator. After masking the noninsulating part of the upper electrode substrate with masking tape (50 $\mu \mathrm{m}$ thickness), the adhesive was applied with a spatula and dried. Finally, the insulating layer was left by removing the masking tape. The thickness of the insulator was measured to be about $14 \mu \mathrm{m}$ using a stylus-type surface profile measuring system (DEKTAK 3ST, Veeco Instruments, Inc.).

The pressure sensor (FlexiForce, A201/4.4 N, Tekscan, Inc., $0.2 \mathrm{~mm}$ thick) uses a pressuresensitive material. The conductance between the two opposing electrodes is changed by applying pressure to the sensor.

The number of wiring lines of the shear force sensor was initially five but was increased to six, and the wiring lines of the pressure sensor were connected to them by soldering. The diameter of the sensors was the same, and both were clamped with an adhesive. The flexibility and bending resistance were maintained by limiting the bonding position of both sensor substrates to three locations, including the sensor head. The wiring pattern of the lower electrode substrate of the shear force sensor was coated with a spray insulating material to prevent the disturbance of the sensor signal when it is touched accidentally during the measurement.

\subsection{Fabrication of measurement system}

We also developed a measurement system to drive the sensor. It was similar to the shear force sensor system ${ }^{(6)}$ but was improved for a three-axis sensor. The configuration of the developed measurement system is shown in Fig. 3. The measurement system consists of a sensor, a control circuit, and PC software. The control circuit is composed of a microcomputer module (LPC4088), a power source using a mobile battery, and electronic components such as operational amplifiers. A sinusoidal AC voltage $(5 \mathrm{kHz}, \pm 200 \mathrm{mV})$ is applied to the sensor, and the current flowing between the sensor electrodes is converted to a voltage, which is then entered into the microcomputer module after voltage amplification, rectification, and smoothing. Here, the peripheral electrodes of the shear force sensor are all set at the ground level. This is to prevent current flowing between the peripheral electrodes as well as crosstalk between the electrodes. The analog signal input to the microcomputer module is converted into digital data by an A/D converter (ADC) in the microcomputer. The digital data is subjected to integration averaging processing in the microcomputer, and then a timestamp is added by the built-in timer. Then, it is transferred in real time via a Bluetooth module to a notebook PC by wireless communication. On the notebook PC, the data sent from the measuring device is further subjected to integration averaging processing by the function of the program, and calibration processing to remove the effects of pressure and temperature is performed. Finally, the corrected data is displayed on the PC screen in real time, and at the same time, the data is stored on the PC as a file. 


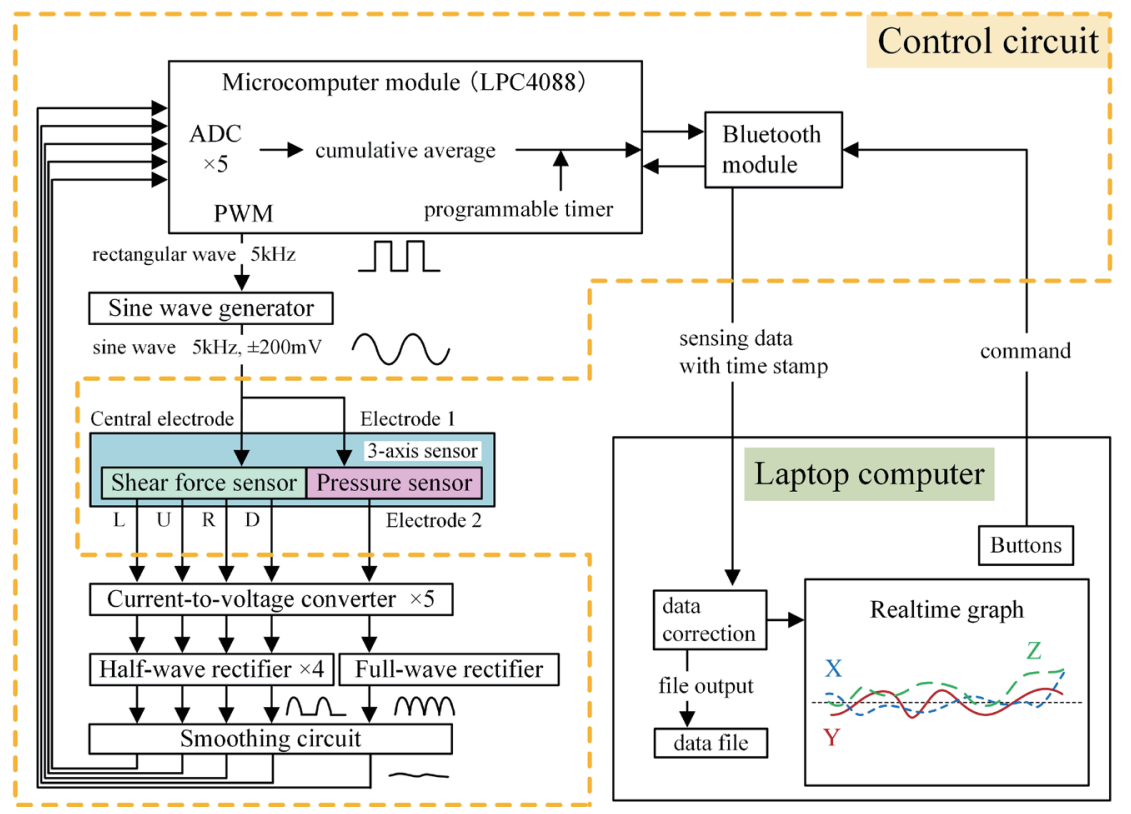

Fig. 3. (Color online) Configuration of measurement system.

Since a shear force sensor uses a liquid electrolyte, under certain conditions, an electrochemical reaction occurs on the electrode surface, which leads to the deterioration of the electrode and an unstable response. To prevent these effects, the use of an aqueous solution is avoided. In addition, a $\pm 200 \mathrm{mV}, 5 \mathrm{kHz}$ sine wave $\mathrm{AC}$ voltage is applied between the electrodes to detect only the electric double-layer charging/discharging current at the electrode surface. ${ }^{(19)}$ Since the pressure sensor does not use a liquid electrolyte, there is no problem even if a DC voltage drives it. However, the same $\mathrm{AC}$ voltage was applied to the pressure sensor to simplify the control circuit as well as to decrease the number of sensor wires. The center electrode of the shear force sensor and one electrode of the pressure sensor (Electrode 1) were made common, and a sinusoidal AC voltage was applied. The four peripheral electrodes of the shear force sensor and the other electrode of the pressure sensor were set at the ground level using an operational amplifier. Here, the output after the current-voltage conversion of the pressure sensor is approximately proportional to the pressure. However, at a small pressure, this output is affected by the forward voltage drop of the diode, so the half-wave rectification circuit cannot be used. Therefore, while the signal from the shear force sensor was half-wave-rectified to simplify the circuit, the signal from the pressure sensor was full-wave-rectified.

\subsection{Calculation process}

For the shear force sensor, the method of calibrating the measured values followed our previous method. ${ }^{(9)}$ When a shear force is applied to the sensor, the rubber ring is distorted and the upper and lower electrode substrates are displaced in the horizontal direction. As a result, the distance between the center electrode and each peripheral electrode changes. Since the 
space between the center electrode and each peripheral electrode is filled with the electrolyte, the current between the electrodes varies with the distance between the electrodes. After that, the voltage obtained by current-voltage conversion, rectification, and smoothing is captured by the AD converter, and the data is transmitted from the control circuit to the laptop computer. The data is stored on the computer and averaged again. The data at this point is called the raw data here. Since there are four peripheral electrodes, $\mathrm{L}, \mathrm{R}, \mathrm{U}$, and $\mathrm{D}$, that indicate the direction, the raw data is acquired simultaneously from the four electrodes. The data correction process was normalized according to the following arithmetic calculation:

$$
\begin{aligned}
& N O R M_{L}=R A W_{L} /\left(R A W_{L}+R A W_{R}+R A W_{U}+R A W_{D}\right), \\
& N O R M_{R}=R A W_{R} /\left(R A W_{L}+R A W_{R}+R A W_{U}+R A W_{D}\right), \\
& N O R M_{U}=R A W_{U} /\left(R A W_{L}+R A W_{R}+R A W_{U}+R A W_{D}\right), \\
& N O R M_{D}=R A W_{D} /\left(R A W_{L}+R A W_{R}+R A W_{U}+R A W_{D}\right),
\end{aligned}
$$

where $R A W_{X}$ and $N O R M_{X}$ are the raw and normalized data corresponding to each electrode, respectively. We previously found that the effect of temperature was almost eliminated by using the sum of each electrode as the denominator. ${ }^{(9)}$ The normalized data was then processed with the following equations and the resultant output is the "sensor response".

$$
\begin{aligned}
& \text { Force }_{x}=k_{x 1}\left(\text { NORM }_{R}-N O R M_{L}\right)+k_{x 2} \\
& \text { Force }_{y}=k_{y 1}\left(N O R M_{U}-N O R M_{D}\right)+k_{y 2}
\end{aligned}
$$

Here, Force $_{x}$ and Force $_{y}$ represent shear forces in the $X$ and $Y$ (unit: N) directions, respectively, and $k_{x 1}, k_{x 2}, k_{y 1}$, and $k_{y 2}$ are correction coefficients (sensor parameters). Among these coefficients, $k_{x 1}$ and $k_{y 1}$ are those representing the characteristics of each sensor because an individual sensor has different characteristics depending on the fabrication conditions. These coefficients are determined from the calibration curve obtained for each sensor using the testing apparatus. The coefficients $k_{x 2}$ and $k_{y 2}$ are determined at the time of zero point correction when the sensor is set in the measurement environment.

However, $k_{x 1}$ and $k_{y 1}$ are set at the unified values (100.0) a priori, since the purpose of this paper is to show the characteristics of the actual sensor. Therefore, the sensor response in the resulting figures has no physical unit, but it is proportional to the shear force or pressure.

The same sine wave AC voltage $( \pm 200 \mathrm{mV})$ is applied between the two terminals for the pressure sensor. This sensor contains pressure-sensing ink, and the resistance changes with pressure. Then, the current flowing between the two terminals is converted into current/ voltage, rectified, and smoothed, and the voltage is captured by the AD converter. After that, it is corrected by the following process and then fed to the computer to be used as the "sensor response". 


$$
\text { Force }_{z}=k_{z 1} R A W_{P}+k_{z 2}
$$

where Force $_{z}$ is the pressure (unit: N), $R A W_{P}$ is the raw data, and $k_{z 1}$ and $k_{z 2}$ are the correction coefficients. $k_{z 1}$ is determined for each sensor from the calibration curve by adding weights, and $k_{z 2}$ is determined at the time of zero point correction when the sensor is set in the measurement environment.

\subsection{Evaluation method}

The sensor response upon the application of vertical force was measured by placing a weight $(100,200,500$, or $1000 \mathrm{~g})$ on the sensor. The sensor response upon the application of horizontal force was measured by applying a horizontal force to the sensor using a laboratory-made testing apparatus $^{(5)}$ as shown in Fig. 4. The sensor was nondestructively fixed between the movable plate and the immovable plate by adjusting the height of the manual stage. The movable plate and tray are connected via a wire so that the shear force is applied to the sensor by placing a weight on the tray. For example, when a $100 \mathrm{~g}$ weight is placed on the tray, a force of $0.98 \mathrm{~N}$ is applied from right to left in Fig. 4(a). As shown in Fig. 4(b), when the top side of the sensor is in contact with the immovable plate and the back side is in contact with the movable plate, a shear force will be applied to the sensor in the $-X$ direction by placing the weight on the tray. In the shear response evaluation, the sensor was held with a force of about $10 \mathrm{~N}$ to fix it, and the weight $(100,200$, or $500 \mathrm{~g})$ was placed on the tray. Owing to the characteristics of the testing apparatus, the load was applied in the negative direction of the sensor $X$ - and $Y$-axes. The baseline of each axis was reset to zero immediately before the start of measurement. Loading and unloading the weight were performed every $60 \mathrm{~s}$.

\section{Results and Discussion}

Figure 5 shows a photo of the produced three-axis load sensor. The total thickness of the sensor was $0.9 \mathrm{~mm}$. Figures 5(a)-5(c) are the whole, magnified, and side views of the sensor, respectively. The $X$-, $Y$-, and $Z$-axes are indicated in Fig. 5(b) to define the directions of the sensor output.

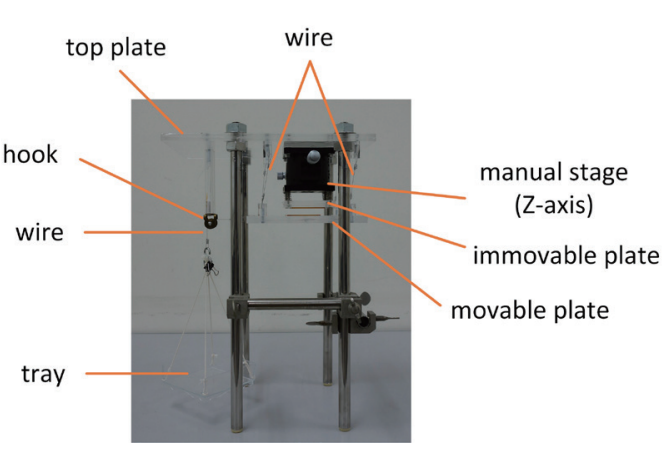

(a)

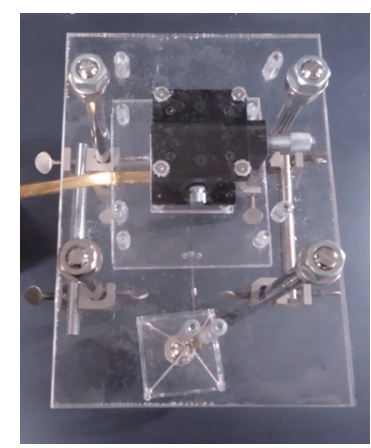

(b)

Fig. 4. (Color online) Testing apparatus for shear force sensor. (a) Side and (b) top views. 


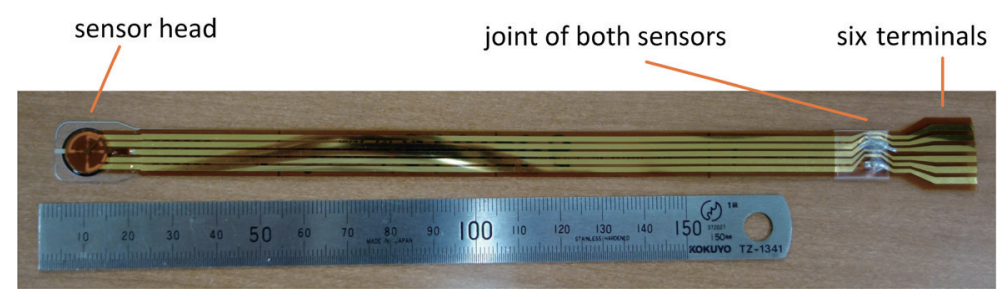

(a)

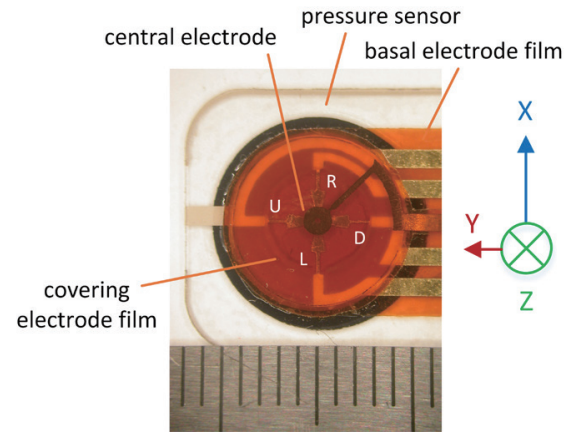

(b)

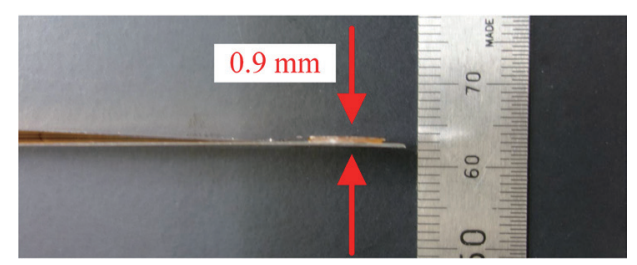

(c)

Fig. 5. (Color online) Three-axis load sensor.

Figure 6 shows the overall view of the measurement system. The major components of the whole system are a three-axis load sensor, a measuring device, a mobile battery for the power supply of the measuring device, and a notebook PC where a program for measurement is running.

Both the pressure sensor and the shear force sensor that make up a three-axis load sensor can be regarded as a variable resistance whose value changes owing to the external force. In the case of the shear force sensor, each of the four peripheral electrodes corresponds to a resistor. Therefore, the response of the control circuit was evaluated by connecting resistors as model sensors. The results are shown in Fig. 7. Figure 7(a) shows the relationship between the conductance of the dummy resistor and the sensor output, and Fig. 7(b) is an enlarged view of part of Fig. 7(a). The horizontal axis shows the conductance of the model resistors, and the vertical axis shows the value read by the ADC in the control circuit. The circular plots $(\bullet)$ correspond to the shear force sensor, and the rectangle plots ( $\mathbf{(})$ correspond to the pressure sensor. For the shear force sensor, the average of the four electrodes was plotted. The leastsquares method was performed within the linear range of less than 0.7 on the vertical axis, and the approximate line was drawn in the graph. The plot corresponding to the shear force sensor is linear but does not pass through the origin. This is because a half-wave rectifier circuit is used. On the other hand, the plot corresponding to the pressure sensor passes through the origin. This is because a full-wave rectifier circuit is used. In the case of a shear force sensor, the conductance can be easily placed in a linear region by adjusting the concentration of the electrolyte. However, in the case of a pressure sensor, the conductance has a wide range owing 


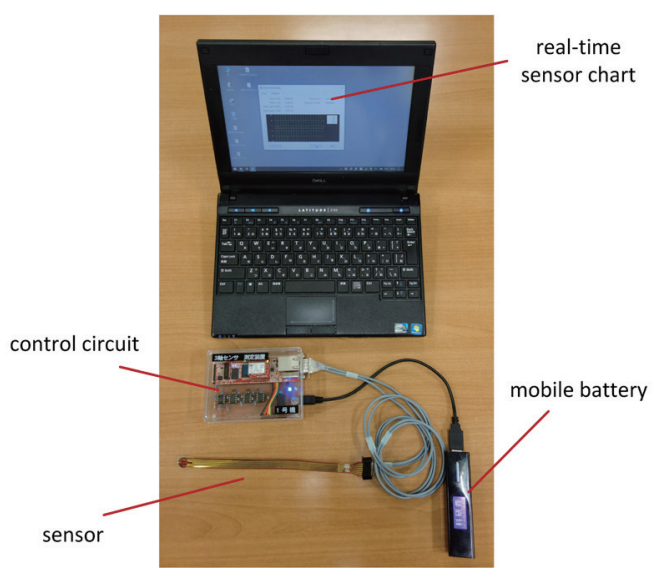

Fig. 6. (Color online) Measurement system for three-axis load sensor.

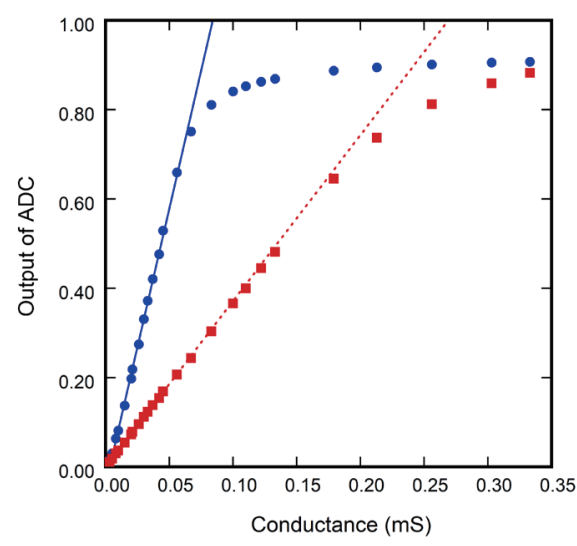

(a)

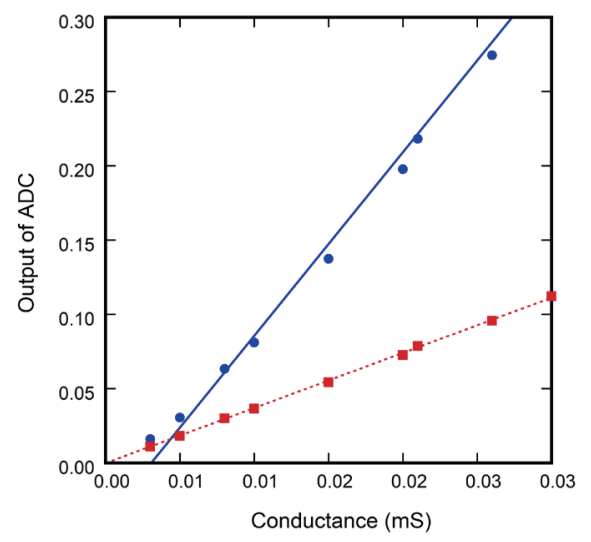

(b)

Fig. 7. (Color online) Relationship between the conductance of model sensors and circuit responses; (a) represents the entire measurement and (b) is a partial enlargement. The solid lines show the fitting of the plots $(\bullet)$ of the average responses of the four model electrodes (L, R, U, and D) and the dashed lines show the fitting of the plots ( of the response of the model pressure sensor.

to the characteristics of the pressure-sensitive material, and when no pressure is applied, the conductance becomes almost zero. For this reason, the latter has to use a full-wave rectifier circuit. As a result, the linearity is ensured in a wide pressure measurement range.

An evaluation experiment was performed by applying vertical and horizontal forces to the sensor. Figure 8 shows the results. In all the graphs of Fig. 8 , the values on the vertical axis have no unit because they are obtained by multiplying a value taken into the ADC by a constant (the same constant in all the graphs). The responses corresponding to the direction unrelated to the load were relatively small in all cases, as shown in Figs. 8(a)-8(c). The gradual response observed after the rapid change is probably mainly due to the creep of silicone rubber and not to the characteristics of the circuit or software. Silicone rubber is used not only for the sensors but for the testing apparatus to improve the adhesion between the sensor and the table. The sensor 


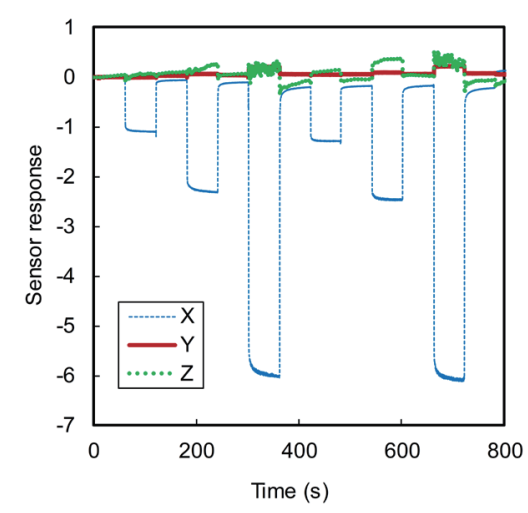

(a)

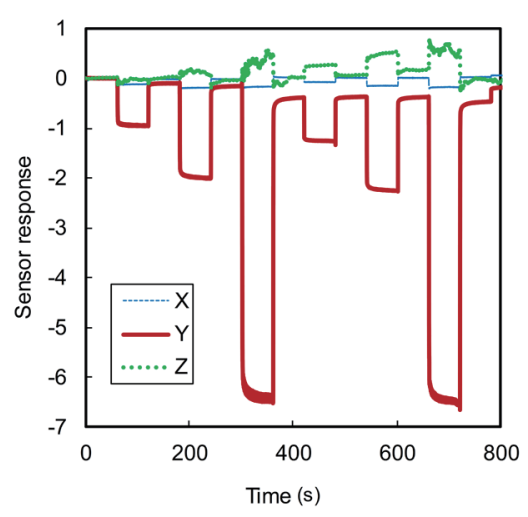

(b)

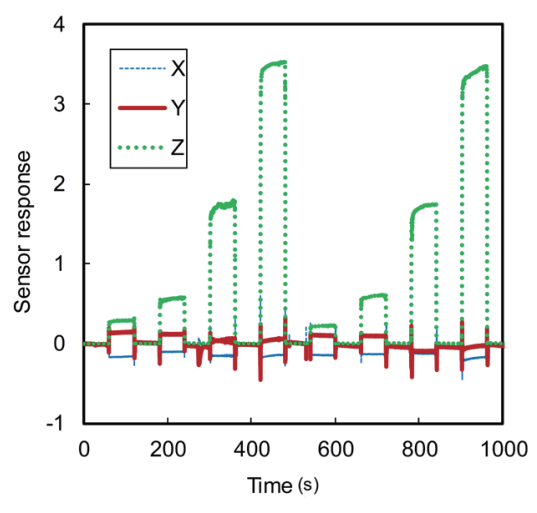

(c)

Fig. 8. (Color online) Sensor response upon the application of load. Force was applied in the (a) $-X$ direction, (b) $-Y$ direction, and (c) $+Z$ direction. The value on the vertical axis is actually 100 times larger than that from the AD converter $(0$ at time 0$)$.

used a $0.5 \mathrm{~mm}$ rubber ring and the testing apparatus used two $0.1 \mathrm{~mm}$ rubber sheets to sandwich the sensor. Therefore, by simply estimating from the thickness ratio, it is considered that $5 / 7$ of the creep may be caused by the sensor.

The axial sensor response to the applied load was calculated from the data in Fig. 8, and the graphs in Fig. 9 were obtained. Specifically, the average response was calculated except for $5 \mathrm{~s}$ immediately before and $10 \mathrm{~s}$ immediately after the placement of the weight. Since the measurement was carried out twice using the same weight, the average of the two responses was reflected at each point. The sensor responses corresponding to the direction of force were almost proportional to the applied load, but those perpendicular to the direction of force were small. These results suggest that the responses corresponding to the different axes were almost independent of each other. Therefore, when using this sensor as a three-axis sensor for the applied measurement, it is sufficient to obtain in advance the proportional coefficient of the load and the response in each axis for each sensor.

According to Ref. 5, when a $0.01 \mathrm{MPa}$ shear force was applied to the palm of the hand, blood flow was halved, and the effective pressure was approximately doubled. Therefore, it is considered that the upper limits of $0.05 \mathrm{MPa}$ for shear force and $0.1 \mathrm{MPa}$ for pressure are appropriate.

The life of the sensor is less than 3 months when exposed to an indoor environment. After this period, we observed a small dent at the center of the sensor, probably due to the evaporation of ethylene glycol (vapor pressure: $6.5 \mathrm{~Pa}$ ) through the polyimide film surface. However, this problem was solved by using tetraethylene glycol with lower vapor pressure $\left(6.2 \times 10^{-3} \mathrm{~Pa}\right)$ as the liquid electrolyte and storing the fabricated sensor in an aluminum-laminated pack. In this case, there was almost no apparent change after at least half a year at room temperature (about $23^{\circ} \mathrm{C}$ ). However, the sensor in this paper used ethylene glycol following our previous article. ${ }^{(9)}$ The graphs were obtained several days after the fabrication of the sensor. 


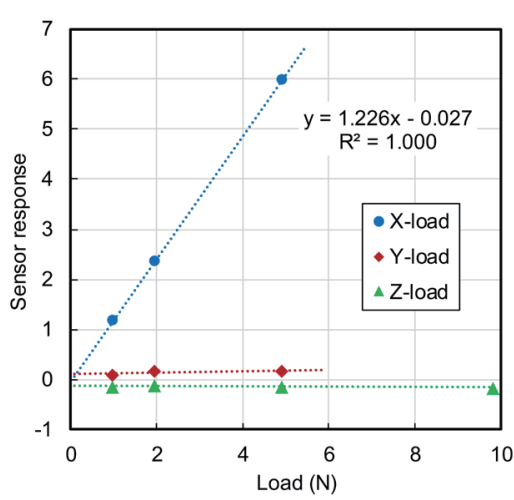

(a)

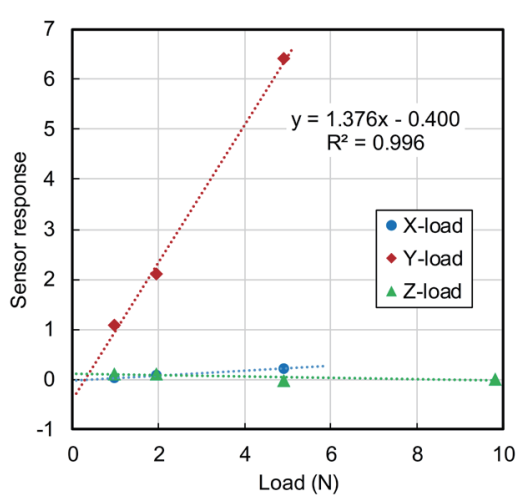

(b)

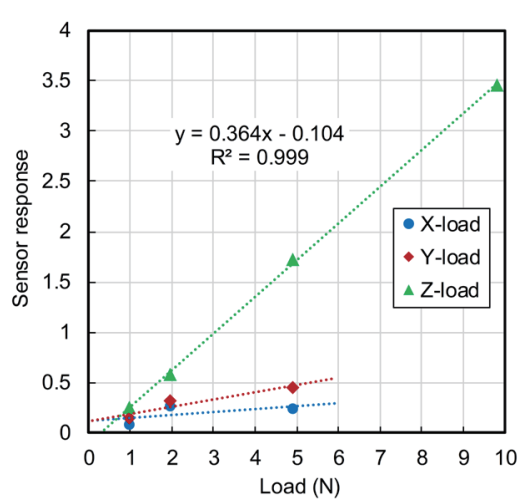

(c)

Fig. 9. (Color online) Sensor response corresponding to each axis upon the application of loads. (a), (b), and (c) are the responses corresponding to the sensor outputs of the $X-, Y$-, and $Z$-axes, respectively.

\section{Conclusions}

We developed a three-axis load sensor and its measurement system. When hybridizing two types of sensors, the number of signal lines was successfully reduced by devising a circuit. Although the thickness was increased by laminating two sensors with different principles, the resultant three-axis sensor became as thin as $0.9 \mathrm{~mm}$. The sensor responses corresponding to the direction of force were almost proportional to the applied load. Moreover, the responses corresponding to different axes were almost independent of each other. As a result, the hybrid sensor can be used as a thin three-axis sensor and fit on even slightly curved surfaces.

\section{Acknowledgments}

This work was supported by JSPS KAKENHI Grant Number JP18K11419.

\section{References}

1 S. M. Reichel: J. Am. Med. Assoc. 166 (1958) 762. https://doi.org/10.1001/jama.1958.62990070004010a

2 S. Dinsdale: Arch. Phys. Med. Rehabil. 55 (1974) 147.

3 D. M. Brienza and M. J. Geyer: Adv. Skin Wound Care 18 (2005) 151.

4 J. E. Grey, S. Enoch, and K. G. Harding: Br. Med. J. 332 (2006) 472.

5 L. Bennett, D. Kavner, B. Lee, and F. Trainor: Arch. Phys. Med. Rehab. 60 (1979) 309.

6 J. S. Akins, P. E. Kar, and D. M. Brienza: J. Rehabil. Res. Develop. 48 (2011) 225.

7 K. Kobara, H. Osaka, H. Takahashi, T. Ito, D. Fujita, and S. Watanabe: J. Phys. Ther. Sci. 26 (2014) 701.

8 T. Kamegaya: J. Phys. Ther. Sci. 28 (2016) 2830.

9 S. Toyama, Y. Tanaka, S. Shirogane, T. Nakamura, T. Umino, R. Uehara, T. Okamoto, and H. Igarashi: Sensors 17 (2017) 1752. https://doi.org/10.3390/s17081752

10 J. E. Sanders and C. H. Daly: J. Rehabil. Res. Dev. 30 (1993) 191.

11 M. Sohgawa, D. Hirashima, Y. Moriguchi, T. Uematsu, W. Mito, T. Kanashima, M. Okuyama, and H. Noma: Procedia Eng. 25 (2011) 627.

12 D. Molnár, A. Pongrácz, M. Ádám, Z. Hajnal, V. Timárné, and G. Battistig: Microelectron. Eng. 90 (2012) 40. https://doi.org/10.1016/j.mee.2011.05.030 
13 H. Takahashi, A. Nakai, N. Thanh-Vinh, K. Matsumoto, and I. Shimoyama: Sens. Actuators A 199 (2013) 43. https://doi.org/10.1016/j.sna.2013.05.002

14 J. Missine, E. Bosman, B. Van Hoe, R. Verplancke, G. Van Steenberge, S. Kalathimekkad, P. Van Daele, and J. Vanfleteren: Sens. Actuators A 186 (2012) 63.

15 T. Iwasaki, T. Takeshita, Y. Arinaga, K. Uemura, H. Ando, S. Takeuchi, M. Furue, E. Higurashi, and R. Sawada: Sens. Actuators A 221 (2015) 1.

16 H. Yousef, J.-P. Nikolovski, and E. Martincic: Procedia Eng. 25 (2011) 128.

17 G. Liang, Y. Wang, D. Mei, K. Xi, and Z. Chen: J. Microelectromech. Syst. 24 (2015) 1510.

18 K. Sasagawa, T. Oyama, K. Tokiyoshi, and K. Yokoyama: JJSEM 13 (2013) s228.

19 S. Toyama, S. Utsumi, T. Nakamura, T. Noguchi, and Y. Yoshida: Sens. Lett. 11 (2013) 442. https://doi. org/10.1166/sl.2013.2740

20 T. Nakamura and S. Toyama: Proc. 24th Jpn. Acad. Prosthet. Orthot. Conf. 25 (2017) 208.

21 S. Shirogane and S. Toyama: Assistive Tech. (2018). https://doi.org/10.1080/10400435.2018.1547333

22 S. Toyama, Y. Tanaka, Y. Ishikawa, and K. Hara: Sens. Mater. 28 (2016) 279. https://doi.org/10.18494/ SAM.2016.1273 